\title{
Who makes it in psychiatry: CV predictors of success in training grades
}

\author{
C. L. E. Katona, Professor of Psychiatry of the Elderly; and M. M. Robertson, \\ Senior Lecturer, University College and Middlesex School of Medicine, \\ Wolfson Building, Riding House Square, London W1N 8AA
}

\begin{abstract}
A recent paper by Lewis (1991) found authorship of a publication to be the only variable that significantly predicted whether applicants for a senior registrar (SR) rotation in psychiatry at a London teaching hospital were shortlisted. We have re-examined curriculum vitae (CV) predictors of shortlisting at SR level within a comparable London teaching hospital rotation (University College and Middlesex Medical School, UCMSM), and extended the study to examine applicants for the corresponding SHO/registrar (REG) rotation. We have also compared the two groups in terms of demographic data and academic achievements. Our intention was to replicate Lewis' findings, and to examine in more detail the possible predictive effects of academic achievement and ethnicity.
\end{abstract}

\section{The study}

There were 32 applicants for three posts on the SR rotation and 27 for nine (five registrar and four SHO) posts on the REG rotation. We were both on the SR appointments committee, and one of us (CLEK) was also on the REG committee. Our data collection took place prior to shortlisting. Since the great majority (18/27) of candidates for the REG rotation were shortlisted, we analysed REG data by appointment (in terms of being offered a post) rather than by shortlisting success. SR shortlisting was carried out by a member of the personnel department using a points system to combine individual committee members' shortlists. Candidates who were placed on the shortlist at any time (i.e. including those who withdrew and those who replaced them on the final shortlist) were included as shortlisting successes in the analysis. Variables examined were gender, age, marital status, birth in the UK, English name, attendance at a British medical school, teaching hospital rotation, having spent more than one year in another specialty, having a degree (other than MB, BS or equivalent), having a higher degree, authorship of any publication(s), authorship of data containing publication(s), number of research projects listed, length of CV, months post-MRCPsych (SRs only), and being a local trainee (SRs only). We undertook statistical analysis using the Easistat (Curtis, 1990) package, and Wilcoxon and $\chi^{2}$ tests as appropriate. Only statistically significant findings are reported below.

\section{Findings}

\section{(a) SR applicants}

(i) Description. The demographic and academic characteristics of the SR applicants are summarised in Table I.

(ii) Predictors of shortlisting. Having a publication, being born in the UK, having an English name, and having received British undergraduate training were all significantly associated with being shortlisted. Comparisons between the shortlisting successes and failures are summarised in Table II

(iii) Significant relationships between predictors. Not surprisingly, there were highly significant relationships between being born in the UK and both having an English name $(P<0.0001)$ and having attended a British undergraduate medical school $(P<0.0001)$. Having an English name was also significantly associated with having had a British undergraduate education $(P<0.001)$. There was a more modest $(P<0.05)$ association between having a data containing publication and having been to a British undergraduate medical school.

\section{(b) REG applicants}

(i) Description (and comparison with $S R$ applicants). Demographic and academic characteristics are presented in Table I. REG applicants were much $(P<0.001)$ less likely to have published anything and had started or completed less than half the number of research projects compared with the SRs $(P<0.0001)$. They also differed significantly from their SR counterparts in length of $\mathrm{CV}$ which averaged nearly three pages shorter $(P<0.0001)$.

(ii) Predictors of appointment. Comparative characteristics of those REG applicants who were or were not appointed are shown in Table II. Both academic achievement and ethnic background associated significantly with shortlisting success. The single most 
TABLE I

Descriptive characteristics of applicants for senior registrar and registrar posts and comparisons between the two groups

\begin{tabular}{lccc}
\hline & Senior registrar & Registrar & $P$ \\
\hline Sex (M/F) & $28 / 4$ & $18 / 8$ & NS \\
Age(years; mean, s.d., range) & $33.9 ; 4.0 ; 28-43$ & $32.3 ; 6.4 ; 25-48$ & NS \\
Marital status(Married/not married) & $18 / 10$ & $11 / 11$ & NS \\
Born in UK (Yes/No) & $16 / 16$ & $11 / 15$ & NS \\
English Name(Yes/No) & $14 / 18$ & $10 / 17$ & NS \\
GB Undergraduate(Yes/No) & $19 / 13$ & $14 / 13$ & NS \\
$>1$ year in other specialty(Yes/No) & $12 / 20$ & $13 / 13$ & NS \\
Any degree(Yes/No) & $12 / 20$ & $7 / 20$ & $<0.001$ \\
Any publication(Yes/No) & $21 / 11$ & $6 / 21$ & $<0.05$ \\
Data containing publication(Yes/No) & $12 / 20$ & $4 / 23$ & $<0.0001$ \\
Number of research projects(mean, s.d., range) & $3.3 ; 1.6 ; 0-6$ & $1.4 ; 2.2 ; 0-10$ & $<0.0001$ \\
Length of CV (mean, s.d., range - pages) & $6.6 ; 2.4 ; 0-11$ & $3.9 ; 1.9 ; 0-9$ & \\
\hline
\end{tabular}

TABLE II

Comparisons between successful and unsuccessful candidates

\begin{tabular}{|c|c|c|c|c|}
\hline & \multicolumn{2}{|c|}{ Senior registrar candidates } & \multicolumn{2}{|c|}{ Registrar candidates } \\
\hline & Shortlisted & Not shortlisted & Appointed & Not appointed \\
\hline $\operatorname{Sex}(M / F)$ & $10 / 2$ & $18 / 2$ & $5 / 4$ & $10 / 4$ \\
\hline Age (Years: mean) & 29.0 & 35.0 & 32.8 & $34.6^{*}$ \\
\hline \multicolumn{5}{|l|}{ Marital status } \\
\hline (Married/single) & $8 / 4$ & $10 / 6$ & $2 / 6$ & $9 / 2^{*}$ \\
\hline Born in UK & $9 / 3$ & $7 / 13^{*}$ & $5 / 3$ & $3 / 12$ \\
\hline English name & $8 / 4$ & $6 / 14^{*}$ & $4 / 5$ & $3 / 12$ \\
\hline GB undergraduate & $10 / 2$ & $9 / 11^{*}$ & $8 / 1$ & $3 / 12+$ \\
\hline$>1$ year in other speciality & $2 / 10$ & $10 / 10$ & $2 / 7$ & $9 / 5^{*}$ \\
\hline Any degree & $6 / 6$ & $6 / 14$ & $5 / 4$ & $2 / 13^{*}$ \\
\hline Any publication & $12 / 0$ & $9 / 11^{* *}$ & $1 / 8$ & $3 / 12$ \\
\hline Data containing publication & $9 / 3$ & $3 / 17 \dagger$ & $1 / 8$ & $2 / 13$ \\
\hline \multicolumn{5}{|l|}{ Research projects } \\
\hline (mean number) & 3.8 & 2.9 & 1.3 & 1.3 \\
\hline \multicolumn{5}{|l|}{ Length of CV } \\
\hline (mean number pages) & 6.7 & 6.5 & 4.0 & 3.7 \\
\hline Months post MRCPsych & 24.5 & 20.1 & - & - \\
\hline
\end{tabular}

All categories Yes/No unless specified

${ }^{*} P<0.05,{ }^{* *} P<0.01,+P<0.001$.

discriminating characteristic of appointees was having had a British undergraduate medical training $(P<0.001)$. Youth $(P<0.02)$, single status $(P<0.05)$, having a first degree $(P<0.05)$ and not having spent more than a year out of psychiatry $(P<0.05)$ were also associated with success.

(iii) Significant relationships between predictors. Youth was associated with attendance at a British undergraduate medical school $(P<0.01)$, not having spent more than a year out of psychiatry $(P<0.01)$, and single status $(P<0.05)$. Being single was also associated with attendance at $a$ British undergraduate medical school $P<0.02$ ).

\section{Comment}

Our results confirm the findings of Lewis (1991) that having a publication of any kind is significantly associated with being shortlisted for an SR interview. Unlike Lewis, however, we have demonstrated that achievement of one or more data-containing publications is a more discriminating predictor. In addition to these purely academic attributes, British birth, name and education are associated with shortlisting success. Items within these clusters of attributes are, not surprisingly, closely related but we have also found that the most discriminating items within the 
two clusters (British medical school and data containing publication) were not independent of each other. This provides at least some reassurance (particularly in the light of the recent concern expressed by Mbubaegbu (1992)) that purely ethnic bias was not responsible for our findings.

We were surprised to find so few demographic differences between SR and REG applicants, although predictable differences in academic achievements were present. In particular, REG applicants were hardly younger than SR applicants. This may reflect a subgroup of relatively old and unsuccessful REG applicants, since age was associated with nonappointment to REG posts. Indeed younger age was significantly associated with most of the other predictors of REG success (single status, British undergraduate education and not having spent more than a year out of psychiatry). It was reassuring that, even at REG level, an academic achievement (having a first degree) was positively associated with appointment.
Our results are based on a relatively small sample, and we may be accused of not achieving full independence in analysis since we were involved in the appointment process. What career advice, with these provisos, do our findings enable us to offer aspiring psychiatrists? We are reluctant to suggest recourse to celibacy or name change by deed poll. Trainees do, however, appear well advised to publish (preferably with data) or be damned, and to start young. Members of appointments committees should also become still more aware of their commitment to equal opportunities and their vulnerability to prejudice.

\section{References}

CurTis, D. (1990) Easistat(V.1.2)Oxford: ARCPublications.

LEWIS, S. (1991) The right stuff? A prospective controlled trial of trainees' research. Psychiatric Bulletin, 15, 478-480.

Mbubaegbu, C. (1992) Racial discrimination in registrar appointments. British Medical Journal, 304, 1694.

\section{Trainees' forum}

\section{Psychiatry and the new NHS}

\section{Judy Harrison, Trainee in Public Health Medicine, Stockport Health Authority, Bramhall Moor Lane, Hazel Grove, SK 7 SAB}

The NHS and Community Care Act 1990 has been described as the biggest shake-up to the NHS since its inception. Most psychiatrists will by now be familiar with the new arrangements for community care, but what are the implications of the structural changes in the NHS for psychiatry?

\section{Separation of purchaser and provider}

One of the key changes has been to separate the task of providing care from that of commissioning or purchasing care. The composition of each side of this organisational divide, and the balance of power between the two is as yet unclear, but ground rules are beginning to emerge.

\section{(a) Purchasing authorities}

The broad remit of purchasing authorities is to assess the health needs of their resident populations, using a variety of epidemiological and survey methods, and to purchase services accordingly.

Although there has been some delay in implementation, it is intended that the resources made available to purchasers will be allocated according to the structure and needs of the population in question. This represents a significant shift from the existing pattern of funding based on previous activity levels.

The organisations most clearly identified with the purchasing function so far have been the District Health Authorities. The exact composition of their purchasing teams varies but will usually include a 